\title{
ERRATUM: AN UPPER BOUND FROM HELIOSEISMOLOGY ON THE STOCHASTIC BACKGROUND OF GRAVITATIONAL WAVES (2014, ApJ, 784, 88)
}

\author{
Daniel M. Siegel ${ }^{1}$ and Markus Roth ${ }^{2}$ \\ ${ }^{1}$ Max Planck Institute for Gravitational Physics (Albert Einstein Institute), Am Mühlenberg 1, D-14476 Potsdam-Golm, Germany \\ ${ }^{2}$ Kiepenheuer Institut für Sonnenphysik, Schöneckstr. 6, D-79104 Freiburg, Germany \\ Received 2015 July 27; accepted 2015 July 29; published 2015 September 1
}

We would like to point out that prior to this publication several authors have considered excitation of solar/stellar oscillations by gravitational waves. The very idea of employing the Sun as a detector for gravitational waves dates back at least to the discussion on the excitation of a $160 \mathrm{~min}$ solar oscillation by gravitational radiation (Walgate 1983). This discussion triggered a series of papers that refuted this speculative idea (Bonazzola et al. 1984; Kuhn \& Boughn 1984; Carroll et al. 1984; Fabian \& Gough 1984; Kosovichev 1984; Deruelle 1984). Furthermore, Boughn \& Kuhn (1984) and, more recently, Khosroshahi \& Sobouti (1997) studied the excitation of solar/stellar oscillations by gravitational waves. While Khosroshahi \& Sobouti (1997) focused on computing energy absorption cross sections for polytropic models and did not report upper limits on a background of gravitational waves, Boughn \& Kuhn (1984) did present such limits (as the only ones of the aforementioned authors) assuming an upper bound on the mean squared velocity of a few solar g- and p-mode oscillations. The latter fact was not pointed out in the published version of this paper and we would like to correct this here. For ease of comparison, Figure 1 shows the upper limits of Boughn \& Kuhn (1984) together with the upper bounds of the published paper.

The upper limits presented by Boughn \& Kuhn (1984) were given for individual frequencies in the range of 105.8-2116.7 $\mu \mathrm{Hz}$. The estimate at $105.8 \mu \mathrm{Hz}$ agrees well with our estimates around this frequency. We note that damping rates are uncertain (up to orders of magnitude) in the frequency range $\approx 110-1500 \mu \mathrm{Hz}$ (cf. grey shaded area in Figure 1) and cannot be reliably calculated due to convection-pulsation interactions (Dupret 2002; Belkacem et al. 2009; Chaplin et al. 1997; Houdek 2006; Dupret et al. 2006; see also the published version of this paper). As shown by the present paper, visibility effects can influence the resulting upper bounds by

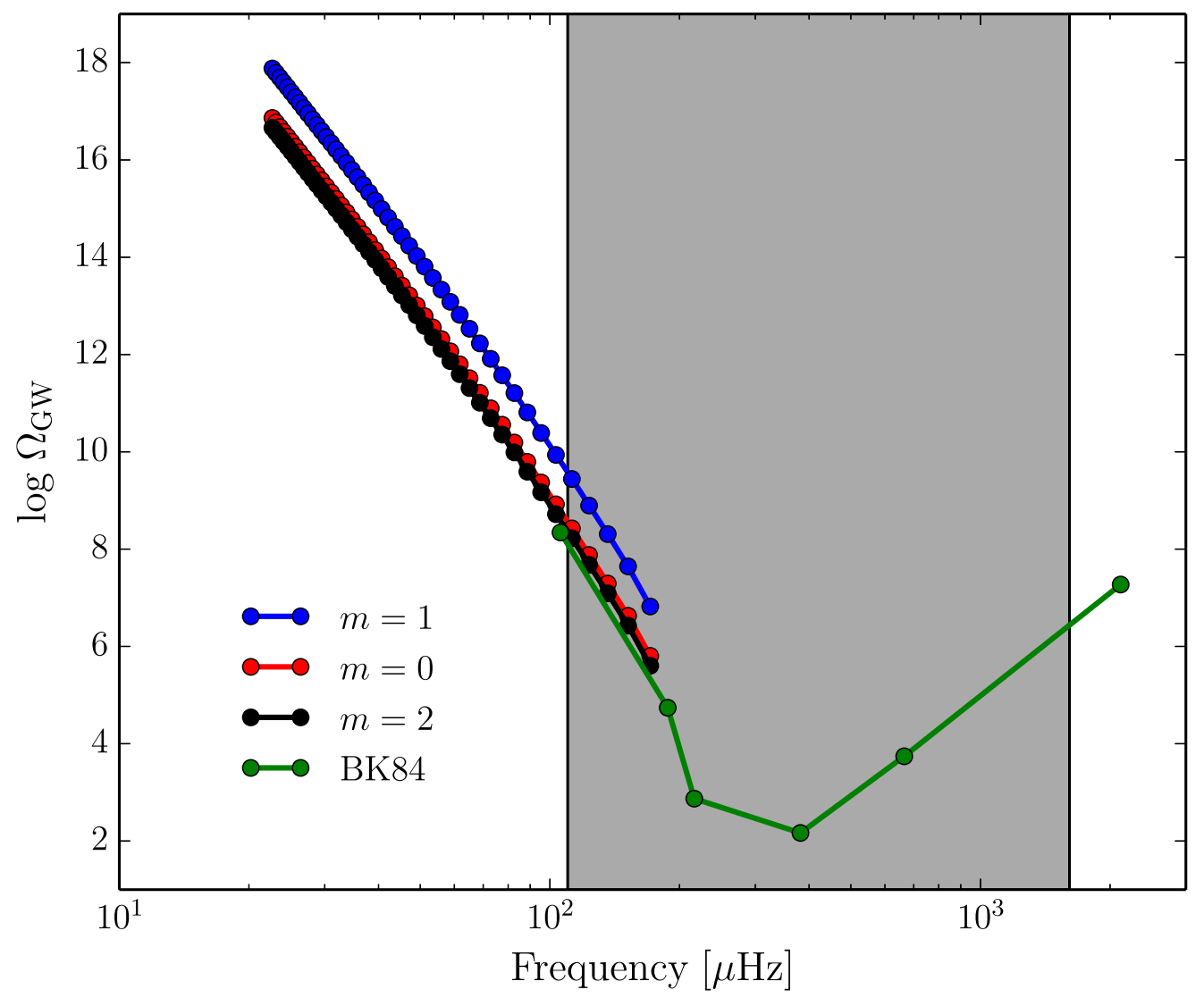

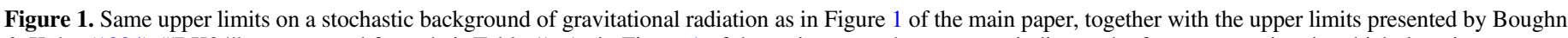

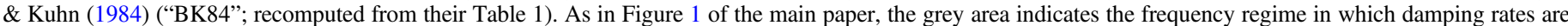
uncertain and cannot be computed reliably (the right end corresponds to the lowest-frequency damping rate measured by Chaplin et al. (1997). 
orders of magnitude as well. It is therefore essential to account for such effects with the best possible accuracy. Our limits represent an improvement with respect to the earlier work of Boughn \& Kuhn (1984) in the sense that we employ updated knowledge on the damping rates and solar modelling, that we base our limits on updated observational data, and that we accurately include mode visibilities.

\section{REFERENCES}

Belkacem, K., Samadi, R., Goupil, M. J., et al. 2009, A\&A, 494, 191

Bonazzola, S., Carter, B., Heyvaerts, J., \& Lasota, J. P. 1984, Nature, 308, 163

Boughn, S. P., \& Kuhn, J. R. 1984, ApJ, 286, 387

Carroll, B. W., McDermott, P. N., Wendell, C. E., \& Shore, S. N. 1984, Nature, 308, 165

Chaplin, W. J., Elsworth, Y., Isaak, G. R., et al. 1997, MNRAS, 288, 623

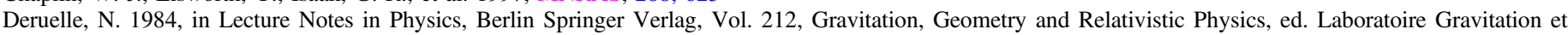
Cosmologie Relativistes, 238

Dupret, M. A. 2002, Bull. Soc. R. Sci. Liège, 71, 249

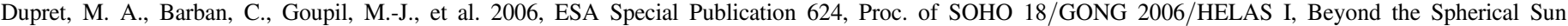
(Noordwijk: ESA), 97

Fabian, A. C., \& Gough, D. O. 1984, Nature, 308, 160

Houdek, G. 2006, ESA Special Publication 624, Proc. of SOHO 18/GONG 2006/HELAS I, Beyond the Spherical Sun (Noordwijk: ESA), 28

Khosroshahi, H. G., \& Sobouti, Y. 1997, A\&A, 321, 1024

Kosovichev, A. G. 1984, SvAL, 10, 190

Kuhn, J. R., \& Boughn, S. P. 1984, Nature, 308, 164

Walgate, R. 1983, Nature, 305, 665 